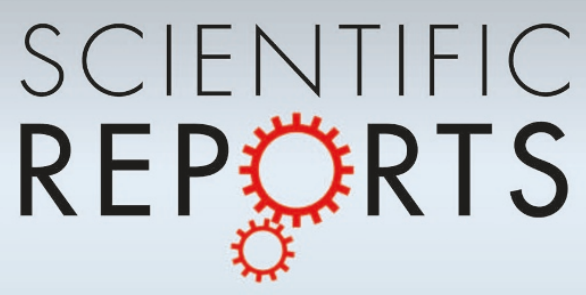

OPEN

SUBJECT AREAS:

PREDICTIVE MARKERS

TRANSLATIONAL RESEARCH

BREAST CANCER

Received

31 March 2014

Accepted

1 December 2014

Published

18 December 2014

Correspondence and requests for materials should be addressed to

K.M. (mukun@sdu. edu.cn)

\footnotetext{
* These authors contributed equally to this work.
}

\section{Over expression of hRad9 protein correlates with reduced chemosensitivity in breast cancer with administration of neoadjuvant chemotherapy}

\author{
Haiqin Yun ${ }^{*}$, Ranran Shi' ${ }^{1}$, Qingrui Yang ${ }^{2}$, Xiaofang Zhang ${ }^{1}$, Yan Wang ${ }^{1}$, Xingchen Zhou' \& Kun Mu' \\ 'Department of Pathology, Shandong University School of Medicine, Jinan 250012, China, ${ }^{2}$ Department of Rheumatology and \\ Immunology, Provincial Hospital Affiliated to Shandong University, Jinan 250021, China.
}

Human Rad 9 (hRad9), part of the Rad9-Hus1-Rad1 complex plays an important role in DNA damage repair as an up-stream regulator of checkpoint signaling, however little is known about its role in response to chemotherapy of breast cancer and whether hRad9 inhibition can potentiate the cytotoxic effects of chemotherapy on breast cancer cells remains to be elucidated. Fifty cases of breast cancer receiving neoadjuvant therapy were collected. All these cases were revised and classified into chemotherapy sensitive (CS) or chemotherapy resistant (CR) group according to the Miller and Payne (MP) grading system. Immunohistochemically, hRad9 positive tumours showed nuclear and/or cytoplasmic staining. hRad9 over-expression was associated with an impaired neoadjuvant chemotherapy response. A significant correlation was found between expression of $\mathrm{hRad} 9$ and Cyclin D1. In vitro, hRad9 was knocked down using siRNA in breast cancer cell line MCF-7 and MDA-MB-231. Deregulated expression of Rad9 accompanied by down expression of chk1 enhanced the sensitivity of human breast cancer cells to doxorubicin. Our work suggests that hRad9 might be a potential predictor for the response to chemotherapy in patients with breast cancer and its clinical value as a target for improving chemosensitivity needs further exploration.

$\mathrm{B}$ reast cancer is the most common cancer and leading cause of cancer-related death amongst women worldwide with a range of overlapping clinical phenotypes that manifest a wide variation in prognosis and outcome $\mathrm{e}^{1,2}$. Chemotherapy has significantly improved the outcome of breast cancer patients. However, chemoresistance becomes a significant obstacle to appropriate treatment of cancer patients and affects the treatment outcome. Chemotherapy can damage DNA in different ways, and activation of the cellular DNA damage repair (DDR) proteins can confer chemoresistance in cancer cells ${ }^{3,4}$. Status of DDR proteins may allow for the prediction of breast cancer sensitivity to chemotherapy and inhibition of the DDR genes or proteins might be effective for increasing tumor chemosensitivity. Recent study showed cells with a high percentage of DNA Damage Repair protein nuclear foci staining correlated with poor tumor response ${ }^{5}$.

The DNA damage checkpoint protein hRad9 was identified as a structural homologue of yeast Schizosaccharomyces pombe rad9 which controls the cell cycle response to DNA damage in Saccharomyces cerevisiae $^{6}$. In response to DNA damage, hRad9 forms a heterotrimeric complex (9-1-1 complex) with Rad1 and Hus1, which is loaded onto DNA damage sites, and facilitates ATR-mediated phosphorylation and activation of Chk1, the latter can cause a G2 cell cycle arrest ${ }^{7-9}$. This process provides extra time for DNA repair before enter into critical phases of the cell cycle. hRad9 gene was mapped to chromosome 11q13.1-13.2 which is close to the frequently amplified gene CCND1 in breast cancer. The role of hrad9 in tumor genesis has been extensively researched. However little is known about its role in response to chemotherapy. Given the roles of hRad9 in DNA Damage Repair, it is reasonable to think that the protein is an important determinant of cell sensitivity to chemotherapeutic drugs that eliminate tumor cells through induction of DNA damage. Inhibiting Rad9 function could sensitize tumor cells to the killing effects of chemotherapeutic treatment.

In this study, we focused on the expression of hRad9 in clinical tumor samples from breast cancer patients who received neoadjuvant chemotherapy which provide a valid clinical model for the evaluation of tumor response. hRad 9 protein expression was performed on both the core biopsy and the corresponding post-neoadjuvant chemotherapy tumor specimen. Our result showed that hRad 9 over expression was associated with an impaired 

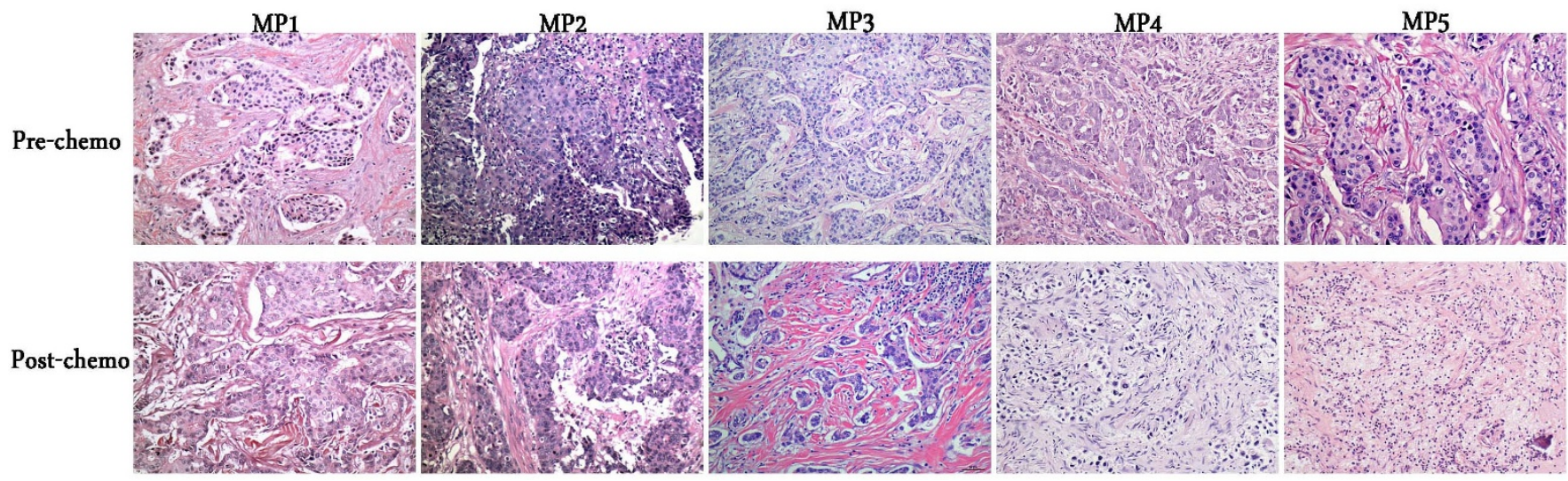

Figure $1 \mid$ Distinct morphological changes in breast cancer specimens after neoadjuvant chemotherapy. According to MP grading system, examples of tumors are shown with predominant morphological changes as MP 1 (No change and no reduction in overall cellularity), MP 2 (A minor up to 30\% loss of tumor cells, but overall cellularity still high), MP 3 (Between an estimated 30\% and 90\% reduction in tumor cells), MP 4 (A marked $>90 \%$ loss of tumor cells such that only small clusters or widely dispersed individual cells remain), MP 5 (No malignant cells identifiable in sections from the site of the tumor; only containing foamy macrophages and vascular fibroelastotic stroma). All images original magnification $\times 200$, haematoxylin and eosin.

neoadjuvant chemotherapy response. In Vitro, We found Silencing hRad9 expression by RNA interference accompanied by down expression of chk1 enhances the sensitivity of human breast cancer cells to doxorubicin. Our work suggests that hRad9 might be a potential predictor for the response to chemotherapy in patients with breast cancer and its clinical value as a target for improving chemosensitivity needs further exploration.

\section{Results}

Over expression of hRad9 in chemoresistant breast carcinomas. Fifty cases of breast cancer receiving neoadjuvant chemotherapy were collected. According to MP grading system, 15 cases were chemotherapy sensitive with more than $30 \%$ loss of tumor cells, while 35 cases showed an impaired neoadjuvant chemotherapy response with a minor up to $30 \%$ loss of tumor cells ${ }^{10}$. Figure 1 shows different morphology appearance response to neoadjuvant chemotherapy using MP grading system.

hRad9, chk1 and cyclin D1 expression were detected by IHC using specific antibodies on both the pre-chemotherapy biopsies and the paired post-chemotherapy tumors. Nucleus staining for hRad9, chk1 and cyclin D1was regarded as positive. hRad9 showed homogeneous nucleus or nucleus/cytoplasm expression with variable intensities in $70 \%$ core biopsy breast specimens. Positive expression rates of chk1 and cyclin D1 in biospy breast cancer tissues were $42 \%$ and $50 \%$, respectively. Figure 2 shows different immunohistochemistry of hRad9, cyclin D1 and chk1 between chemoresistant and chemosensitive carcinomas.

When comparing hRad9 expression with patient's chemotherapy response, a strong correlation was found with chemotherapy resistance $\left(\chi^{2}=40.9, \mathrm{P}=0.000\right.$, table 1$)$. No significant correlations were found between hrad9 expression and other clinicopathological factors including tumor size, lymph node involvement, cell proliferation index, ER, PR and C-erbB2 status. A significant correlation was also found between chk1 over expression and impaired patient's chemotherapy response $\left(\chi^{2}=5.4, \mathrm{P}=0.021\right.$, table 1$)$. By bivariate analysis, a positive correlation was found between hRad9 and chk1 $(\mathrm{R}=$ $0.327, \mathrm{P}=0.020$, table 2$)$, hRad9 and cyclin $\mathrm{D} 1(\mathrm{R}=0.393, \mathrm{P}=$ 0.005 , table 2).

Having defined the close relationship between increased expression of hRad9 and impaired chemotherapy response, we are further interested in clarifying whether the expression level of hRad9 varies before and after neoadjuvant chemotherapy. By Comparing the

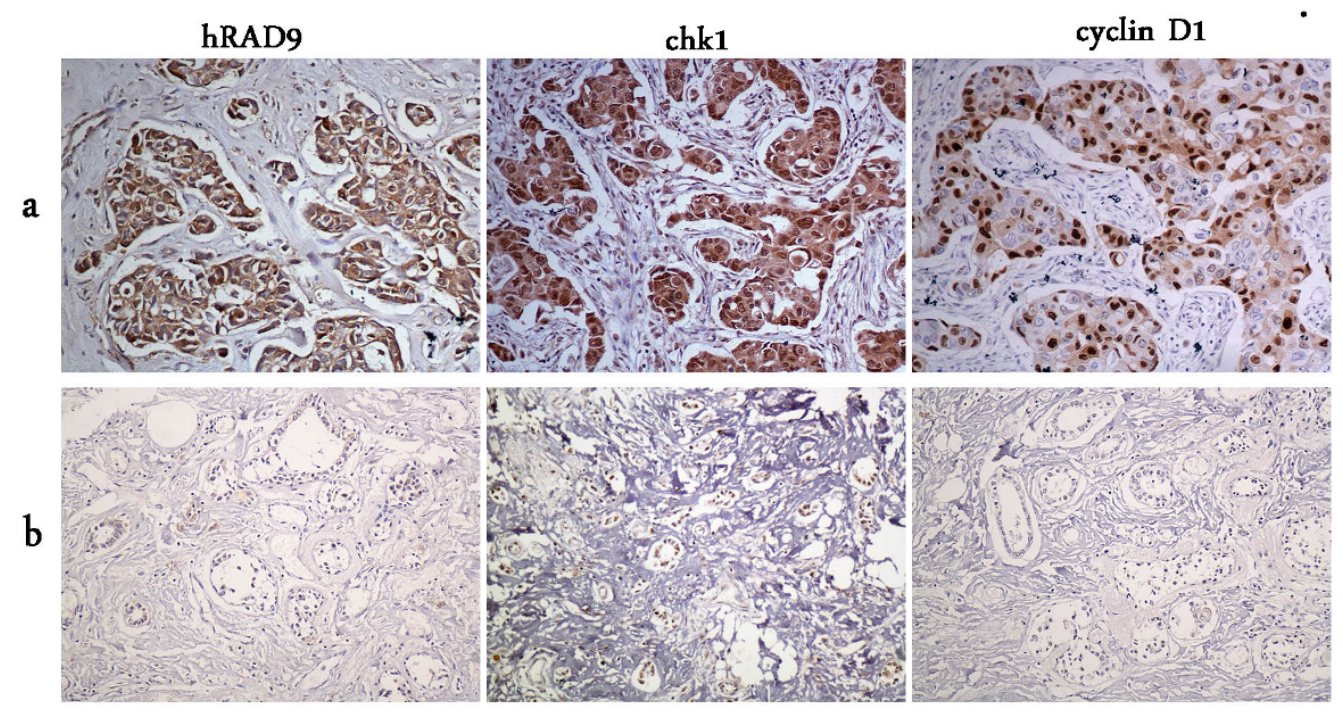

Figure $2 \mid$ Immunohistochemistry of hRad9, cyclin D1 and chk1 in breast carcinoma. Positive expression of hRad9, chk1 and cyclin D1 in chemoresistant breast carcinoma (a) comparing with that of negative expression in chemosensitive carcinoma (b). 
Table 1 | hRad9 and chk 1 expression in relation to neoadjuvant chemotherapy response

\begin{tabular}{|c|c|c|c|c|c|c|}
\hline Expression & \multicolumn{2}{|c|}{ chemotherapy resistant; } & \multicolumn{2}{|c|}{ chemotherapy sensitive } & $\chi^{2}$ & $P$ \\
\hline \multicolumn{7}{|l|}{ hRad9 } \\
\hline $\begin{array}{l}\text { High } \\
\text { Chkl }\end{array}$ & 34 & 97.1 & 1 & 6.7 & 40.9 & $0.000 * *$ \\
\hline Low & 11 & 31.4 & 10 & 66.7 & & \\
\hline
\end{tabular}

immunoreactivity of hRad9 between Post-neoadjuvant chemotherapy tumor specimen and Pre-neoadjuvant chemotherapy biopsy specimen, we did not find significant variation for hRad9 expression before and after chemotherapy $(\mathrm{P}=0.571$, table 3$)$. Interestingly, the immunoreactivity for chk 1 protein was significantly increased after neoadjuvant chemotherapy comparing with that of the biopsy specimen $(\mathrm{P}=0.012$, table 3$)$.

Knockdown of hRad9 enhancing sensitivity to doxorubicin. Small interfering RNA targeting hRad9 was transfected into human breast cancer cell lines MCF-7 and MDA-MB-231. To evaluate the efficacy of hRad9-RNAi, mRNA and protein levels of hRad9 were analyzed. Specific knockdown of hRad9 mRNA expression measured by RTqPCR in MCF-7 and MDA-MB-231 breast cell lines after hRad9siRNA in comparison to controls. hRad9-siRNA significantly decreased the expression of hRad 9 mRNA compared with control cells. Western blot analysis showed hRad9 expression was dramatically decreased in silenced versus non-silenced cells (Figure 3).

To explore whether knockdown of hRrad 9 can increase sensitivity to doxorubicin of breast cancer cells. hRad9-siRNA breast cancer cells and control cells were harvested for MTS assay and PI staining after 48 hours exposure to doxorubicin, as described in Materials and methods. Figure 4 shows knockdown of hRad9 enhanced the doxorubicin-induced growth inhibitory effects on MCF-7 and MDA-MB-231 breast cancer cells. Flow cytometry analysis showed apoptosis rate of hRad9-siRNA treated MDA-MB-231 cancer cells was significantly increased by comparison with controls both before and after doxorubicin treatment (Figure 5).

hRad9 is a key participant in the Chk1 activation pathway, and the latter is a protein kinase which regulate the checkpoint response and chemotherapy response. We examined the expression of Chk1 to evaluate whether the chemoresistence role of hRad9 over expression was related to the hRad9/chk1 signaling pathway. Chk1 was markedly weaker in hRad9-silenced breast cancer cells versus nonsilenced cells. This correlation suggests that the hRad9-Chk1 pathway functions in the cells, and hRad9 silencing deteriorated Chk1 function which might partly explain the chk1 associated chemoresistance.

\section{Discussion}

hRad9 plays an important role in the detection and repair of DNA strands injured by chemotherapy. In response to DNA damage, Rad9 forms a 9-1-1 complex with Rad1 and Hus1 that regulates cell cycle arrest at the G2 checkpoint with chk1 activation ${ }^{11-13}$. Chk1 over expression was correlated with chemoresistance and the possible reason may be part of chk1activation which causes G2 cell cycle arrest and protect cancer cells from the harsh anti-cancer treatment ${ }^{14-16}$. Chk1 is a potential target for anticancer therapy and many chk1 inhibitors are under investigation of clinical trials ${ }^{17,18}$. However, hRad9 as chk1's important upstream activator, little is known about its role in response to chemotherapy of breast cancer and whether hRad9 inhibition can potentiate the cytotoxic effects of chemotherapy on breast cancer cells remains to be elucidated. In the present study both in vivo and vitro we show for the first time that hRad9 correlate with breast cancer sensitivity to DNA damage-inducing chemotherapy and down-regulated hRad9 increases cells susceptibility to doxorubicin in human breast cancer.

Numerous studies have reported that hRad9 over expression occurred in various tumors, including lung cancer, breast cancer, thyroid cancer, prostate cancer ${ }^{19-22}$. Cheng et al found that $52.1 \%$ (25/48) of breast tumors had high levels of overexpressed hRad9 mRNA, which correlated with larger tumor size and local recurrence, while Maniwa's study showed accumulation of hRad9 in the nuclei of tumor cells in 33\% of non-small cell lung carcinoma using immunohistochemical analysis ${ }^{19,20}$. In our study we found that hRad9 had a high expression in chemoresistant breast carcinomas. hRad9 was strongly expressed in the nuclei of chemoresistant breast cancer tumor cells and its expression positively correlated with chk1 expression. However in our study, No significant correlations were found between hRad9 expression and other Clinicopathological parameters, such as large tumor size, axillary node metastases and higher proliferation index.

Interestingly, when we compare the expression of hRad9 and chk1 protein in pre-neoadjuvant chemotherapy biopsy specimen and its corresponding post-neoadjuvant chemotherapy tumor specimen, we found a significant increase of chk1 protein expression, while no significant variation of hRad9 expression was found. It suggests that hRad9 protein over expression might contribute to the innate resistance of tumor cells response to chemotherapy, while chk1 might play an important role on acquired resistance.

Gene amplification or DNA methylation of hrad9 can at least in part account for the high levels of the protein observed ${ }^{20,22}$. hRad9 gene was mapped to chromosome 11q13.1-13.2 which is close to the

Table 2 | Expression of hRad9 in relation to chk1 and cyclin D1

\begin{tabular}{|c|c|c|c|c|}
\hline \multirow[b]{2}{*}{ hRad9 } & \multicolumn{2}{|c|}{ Chkl } & \multicolumn{2}{|c|}{ CyclinD1 } \\
\hline & Low & High & Low & High \\
\hline Low & 10 & 5 & 12 & 3 \\
\hline High & 11 & 24 & 13 & 22 \\
\hline$R$ & \multirow{2}{*}{\multicolumn{2}{|c|}{$\begin{array}{l}0.327 \\
0.02 *\end{array}$}} & \multirow{2}{*}{\multicolumn{2}{|c|}{$\begin{array}{l}0.393 \\
0.005^{* *}\end{array}$}} \\
\hline$P$ & & & & \\
\hline
\end{tabular}


Table 3 | Comparison of the immunoreactivity of hRad9 and chk1 between pre-neoadjuvant chemotherapy biopsy and post-neoadjuvant chemotherapy tumor specimen

\begin{tabular}{llc} 
Tumor specimen & \multicolumn{2}{c}{ Mean intensity score } \\
\cline { 2 - 3 } & hRad9 & Chk1 \\
\hline Pre-chemotherapy & 1.43 & 1.15 \\
Post-chemotherapy & 1.51 & 1.54 \\
$P$ & 0.571 & $0.012^{*}$ \\
\hline${ }^{* P}<0.05$ by statistical analysis of Paired Samples Test. & & \\
\hline
\end{tabular}

a

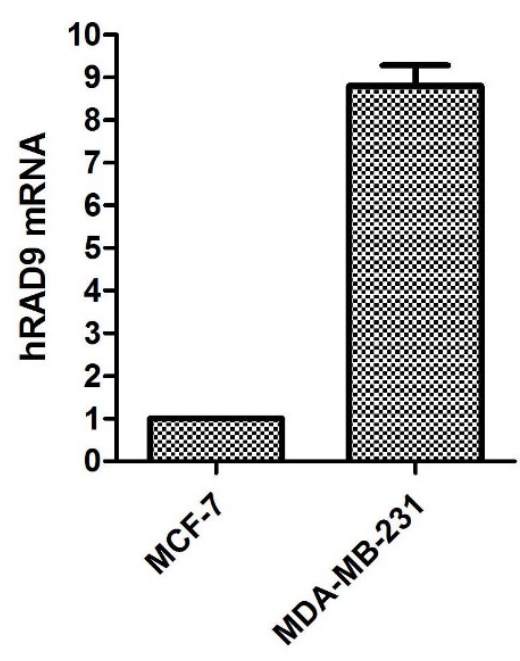

b
C

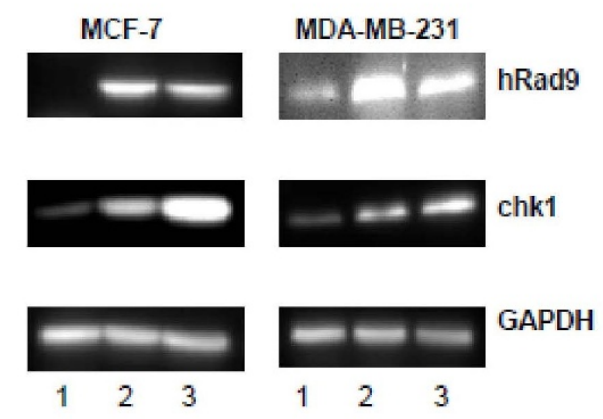

1: siRNA-hRad9 2: Scrambled control 3: Blank control

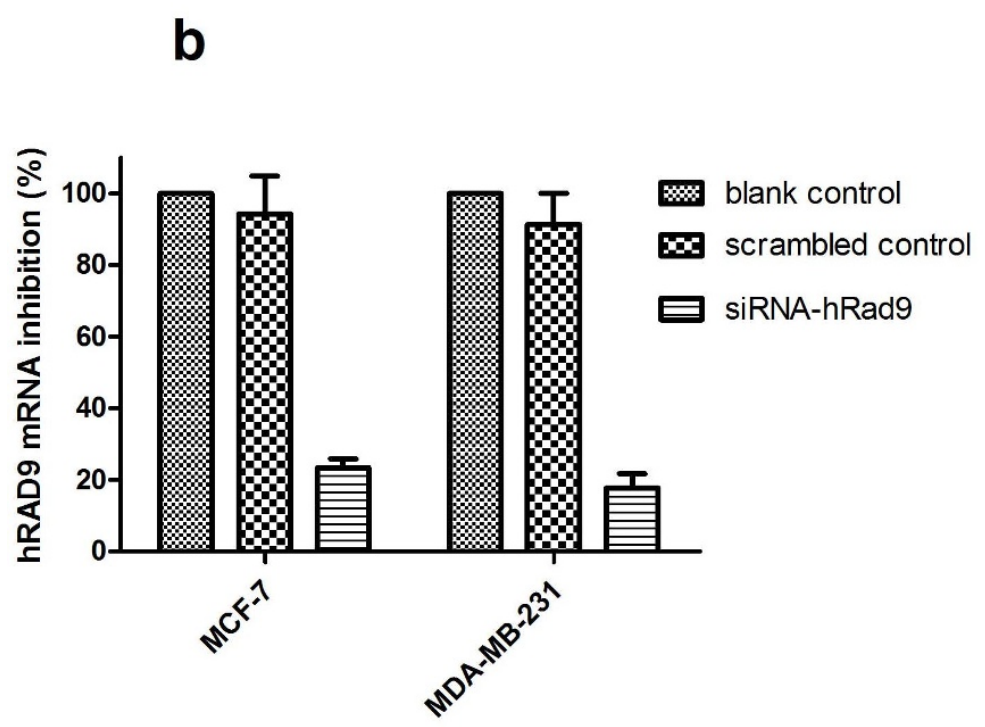

Figure 3 hRad9 is deregulated in MCF-7 and MDA-MB-231 breast cancer cells after hRad9-siRNA treatment. Relative expression of hRad9 mRNA in MCF-7 and MDA-MB-231 breast cancer cells (a). RNA was isolated from cultures after $48 \mathrm{~h}$ in vitro growth and analyzed by RT-qPCR. Internal RT-qPCR control was GAPDH. Results are expressed as fold content in respect to MCF-7 cells (average \pm SD; $n=3$ ). Specific knockdown of hRad9 mRNA expression measured by RT-qPCR in MCF-7 and MDA-MB-231 breast cell lines after hRad9-siRNA in comparison to controls (b).

The $\Delta \Delta \mathrm{Ct}$ method and normalization by comparison with controls were used. Values are means $\pm \mathrm{SD}$ for $\mathrm{n}=3$ assays. Statistical analysis was carried out by Student t-test $(\mathrm{P}<0.05)$. Western blot analysis confirmed the siRNA knockdown of hRad9. The fractionated cell extracts were analyzed with indicated antibodies by immunoblotting (c). hRad9 and chk1 protein expression were deregulated by siRNA knockdown of hRad 9 compared to the scrambled siRNA or blank controls in breast cancer cell lines. 

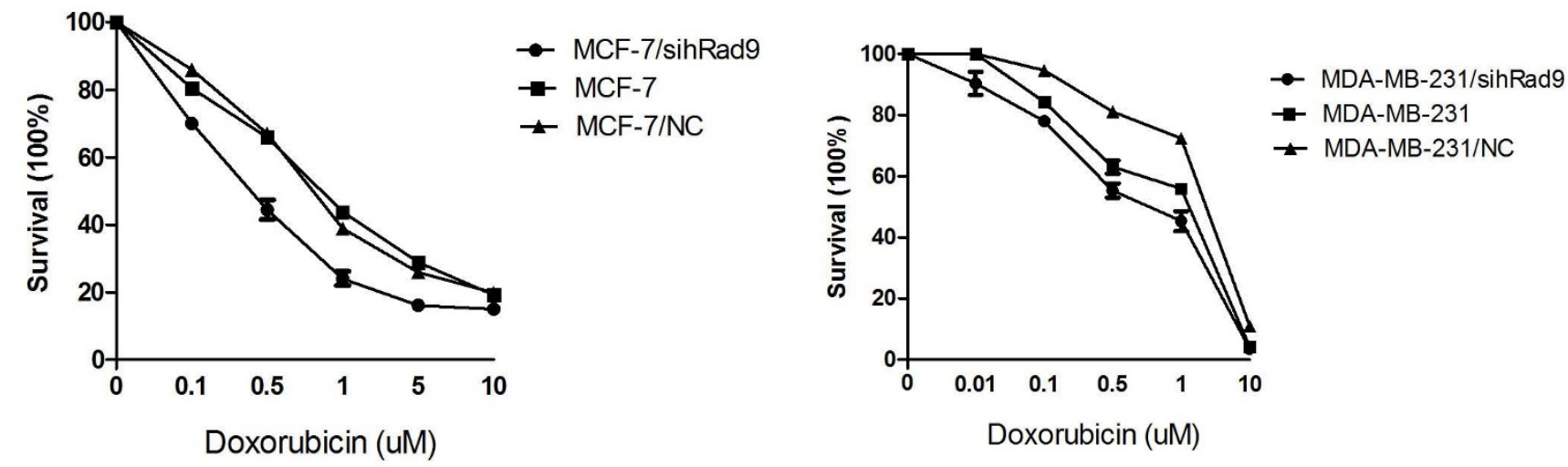

C
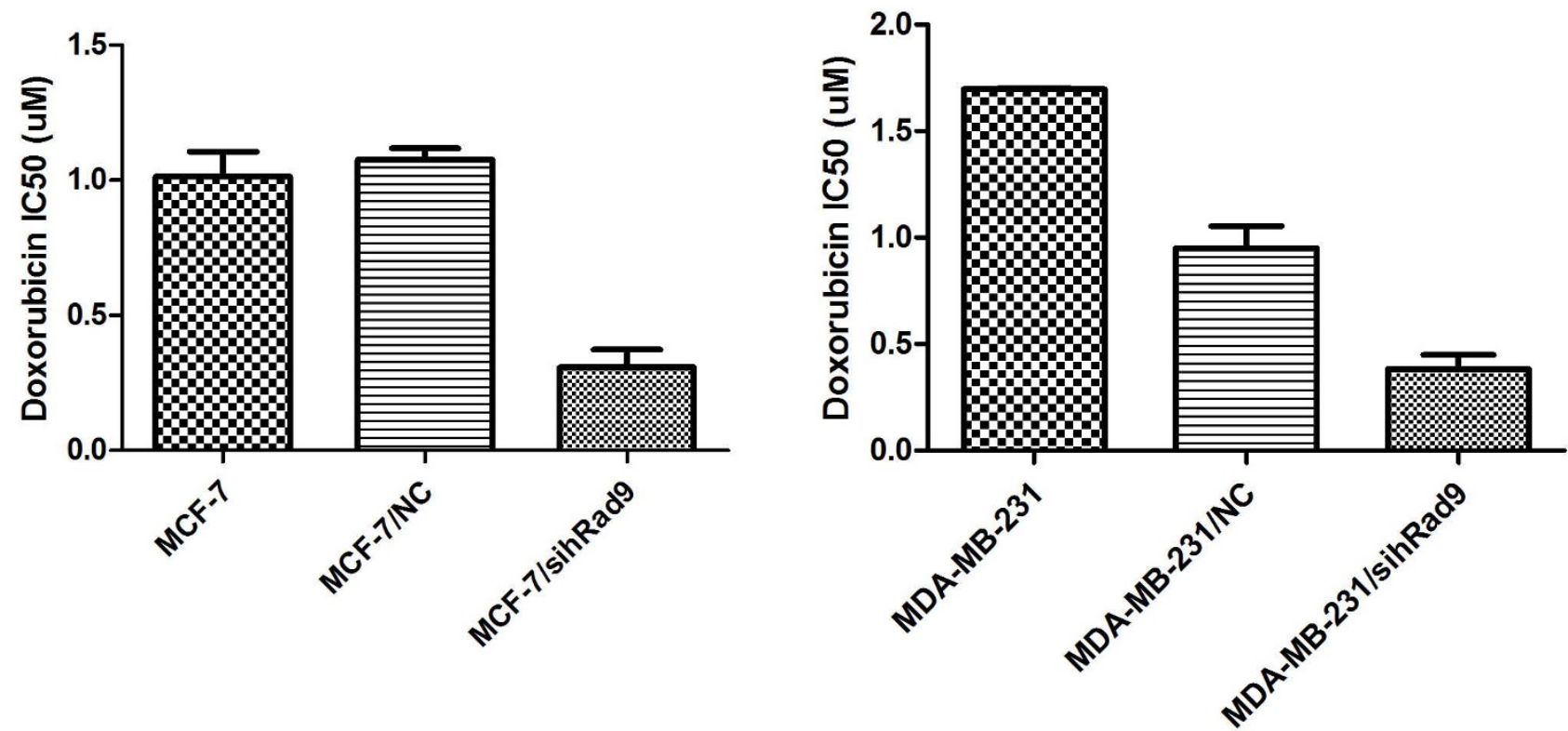

Figure $4 \mid$ Knockdown of hRad9 in breast cancer cells enhances sensitivity to doxorubicin treatment. Knockdown of hRad9 enhanced the doxorubicininduced growth inhibitory effects on MCF-7 (a) and MDA-MB-231(b) breast cancer cells. IC50 value to doxorubicin in hRad9 knockdown breast cancer cells MCF-7 (c) and MDA-MB-231 (d) was significantly decreased comparing with that of the blank controls $(\mathrm{P}<0.001)$ or scrambled negative siRNA ( $\mathrm{P}<0.001$ and $\mathrm{P}<0.005$, respectively, each experiment was performed three times).

cell cycle regulatory gene CCND1. The chromosomal region 11q13 has been proved to be amplified up to $15-20 \%$ of breast carcinomas ${ }^{23}$ and associated with poor prognosis and treatment failure ${ }^{24}$. The 11q13 amplicon pattern is diverse and may consist of several regions that can be amplified independently or together ${ }^{20,23}$. In our previous study, we found $26.2 \%$ (16/61) of breast tumors had CCND1 amplification by in-situ hybridization which was more likely to be associated with tumor progression ${ }^{25}$. In the current study, we found Rad9 protein expression was positively correlated with cyclin D1 protein expression. Our results imply that hRad9 might be involved in the 11q13 amplicon with a resulting protein over-expression parallel in Cheng's report ${ }^{20}$. Considering hRad 9 is frequently amplified in breast cancer, we thought hRad9 might be a potential optimal target for chemotherapy therapy, especially for those patients with 11q13 amplification. 

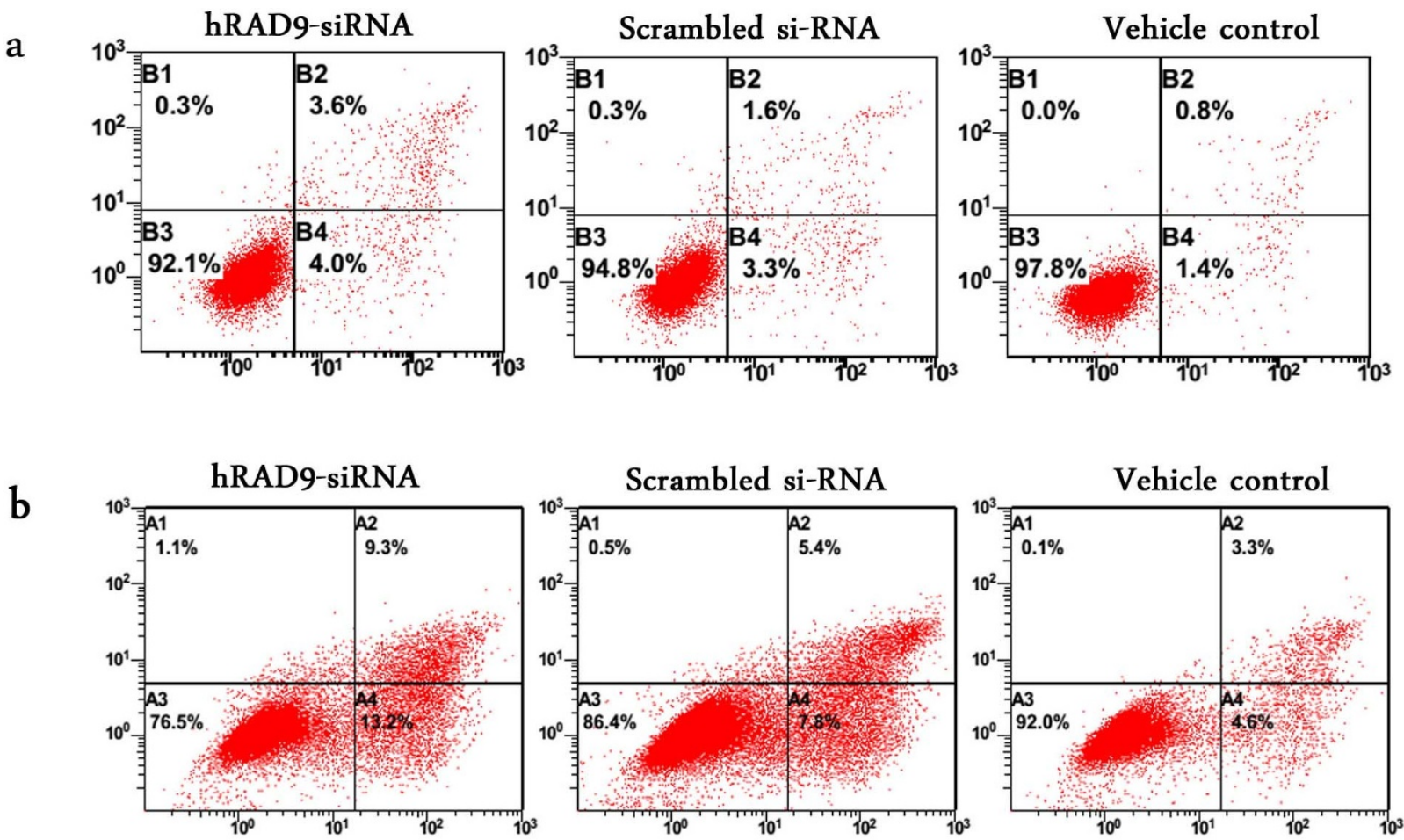

c

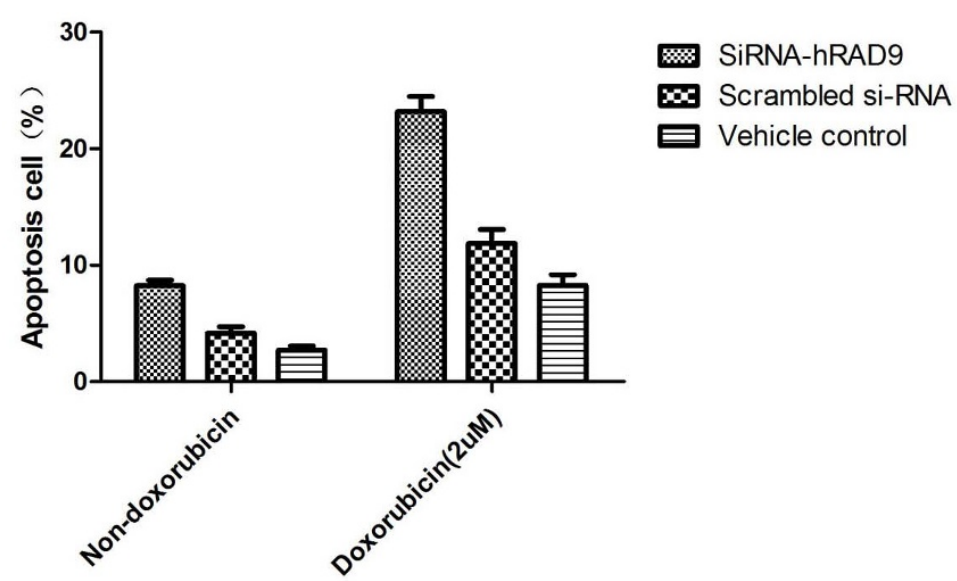

Figure 5 Effects of hRad9-siRNA on apoptosis in MDA-MB-231 breast cancer cells before and after doxorubicin treatment. (a) Flow cytometry images of MDA-MB-231breast cancer cells after hRad9-siRNA treatment. (b) Flow cytometry images of hRad9 knockdown MDA-MB-231 breast cancer cells treated with $2 \mathrm{uM}$ doxorubicin. (c) Flow cytometry analysis showed apoptosis rate of hRad9-siRNA treated MDA-MB-231 cancer cells was significantly increased by comparison with controls both before and after doxorubicin treatment $(\mathrm{P}<0.005$ and $\mathrm{P}<0.001$, respectively, each experiment was performed three times).

In our clinical samples study, hrad9 expression was found positively correlated with chk1 expression. Chk1 is highly expressed in breast cancer especially in triple-negative breast carcinomas ${ }^{26}$. In contrast, its corresponding gene CHEK1 located on 11q24 is often deleted in breast cancer. The precise molecular mechanisms controlling Chk1 activation are not well understood. Moreover, in our previous work we found that 11q13 amplification and 11q-ter deletion are common events in breast cancer, and the two genetic alterations often coexist. As a key upstream regulator of chk1, it might partly explain the chk1 associated chemoresistance for hRad9 gene is frequently amplified while CHEK1 gene is frequently deleted.

Our clinical study showed hRad9 over-expression was associated with an impaired neoadjuvant chemotherapy response. Therefore, we investigated whether hRad9 silencing can potentiate the cytotoxic effects of chemotherapy on breast cancer cells in which hRad9 expression was transiently suppressed. Our qRT-PCR analysis and Western blot results showed a marked decrease of hRad9 and chk1 expression in the Rad9-RNAi treated breast cancer cells. Our results clearly showed hRad9 over expression correlate strongly with an impaired neoadjuvant chemotherapy response and down-regulated hRad9 increases cells susceptibility to chemotherapy in breast cancer cells. The possible mechanism of restoration of sensitivity to chemotherapy could be partly associated with repressed chk1 activation. Since hRad9 plays an important role in DNA damage repair, it is of great interesting to explore whether there are other downstream pathways correlating with chemoresistance in breast cancer.

To our knowledge, this is the first report to explore the role of hRad9 in response to chemotherapy with breast cancer. In vivo, we 
Age at diagnosis

$\leq 45$ years

$>45$ years

Tumor size

$\leq 2 \mathrm{~cm}$

$>2 \mathrm{~cm}$

Lymph node status

Negative

Positive

Grade

1

2

3

ER status

Negative

Positive
PR status

Negative

Positive

HER2 status

Negative

Positive

Proliferation activity

Low $(\mathrm{Ki} 67<15 \%)$

High (Ki67 $\geq 15 \%$ )

Neoadjuvant chemotherapy

AT or ET

CTF

CEF

Other

ER = estrogen receptor; PR = progesterone receptor; HER2 = human epidermalgrowth factor receptor-2, AT = Adriamycin and Taxotere; ET = Epirubicin and Taxotere; CTF = cyclophosphamide,

Taxotere, and 5- fluorouracil; CEF = cyclophosphamide, Epirubicin, and 5- fluorouracil.

focused on the expression of hRad9 in clinical tumor samples from breast cancer patients who accepted neoadjuvant chemotherapy which provide a valid clinical model for the evaluation of tumor response. In vitro, we knocked down hRad9 expression to explore whether hRad9 silencing can potentiate the cytotoxic effects of chemotherapy on breast cancer cells in which hRad9 expression was transiently suppressed. Our work suggests that hRad9 might be a potential predictor for the response to chemotherapy in patients with breast cancer. The data of our study are currently limited, large sample study should be done for its prediction value of hRad9 in different phenotypic groups of breast cancer, such as for tumors with $11 q 13$ amplification. Moreover, the clinical value of $h \operatorname{Rad} 9$ as a target for improving chemosensitivity needs further exploration.

\section{Methods}

Ethics statement. All the protocols dealing with the patients conformed to the ethical guidelines of the Helsinki Declaration and were approved by Shandong University Research Ethics Committee (No. 201301028). Written informed consent was obtained from each patient before participation. This consent procedure was approved by ethics committee.

Tumor samples. 50 breast tumors obtained during surgical resection from Qilu Hospital, Shandong University were used in this study. All the 50 breast carcinomas received at least four cycles of neoadjuvant chemotherapy before surgical resection. Tumor characteristics, including tumor size and axillary lymph node status, were available and collected retrospectively from the files of the Pathology Department of Qilu Hospital, Shandong University (Table 4). Tumor grading was classified according to the 2011 World Health Organization criteria. Response to neoadjuvant chemotherapy was assessed by two pathologists independently, using Miller and Payne (MP) grading system ${ }^{10}$. Grade1: No change or some alteration to individual malignant cells, but no reduction in overall cellularity; Grade2: A minor loss of tumor cells, but overall cellularity still high; up to $30 \%$ loss; Grade3: Between an estimated $30 \%$ and $90 \%$ reduction in tumor cells; Grade4: A marked disappearance of tumor cells such that only small clusters or widely dispersed individual cells remain; $>90 \%$ loss of tumor cells; Grade5: No malignant cells identifiable in sections from the site of the tumor; only vascular fibroelastotic stroma remains, often containing macrophages; however, ductal carcinoma in situ may be present. According to MP grading system, grade 3 to grade 5 tumors were classified into chemotherapy-sensitive (CS) group; grade 1 and grade 2 tumors were regarded as chemotherapy-resistant (CR).

Immunohistochemistry. All the samples were fixed with $10 \%$ (volume/volume) neutral-buffered formalin overnight and then embedded in paraffin. Sections were deparaffinized and subjected to antigen retrieval by autoclave in $0.01 \mathrm{~m}$ EDTA buffer (pH 8.5) for $5 \mathrm{~min}$, followed by incubation in $3 \% \mathrm{H} 2 \mathrm{O} 2$ for 10 min to quench endogenous peroxidase. Primary antibodies for Immunohistochemistry (IHC) included specific antibodies against hRad9 (NBP1-47946, Novus Biologicals, LLC, USA) with a dilution of $1: 1000$, chk1 (NB100-464, Novus Biologicals, LLC, USA) with dilution of $1: 300$ and cyclinD1 (ZA-0101, ZSGB-BIO, Beijing, China) with a dilutin of $1: 30$. Sections were incubated overnight at $4{ }^{\circ} \mathrm{C}$ with primary antibodies diluted in $1 \%$ BSA. Substitution of the primary antibody with PPS served as a negative control. After washed with PBS for 3 times and incubated with secondary antibody (EnVision ${ }^{\mathrm{TM}}$ Detection Systems K5007, Dako Denmark A/S) at room temperature for
$0.5 \mathrm{~h}$, staining was carried out by incubating the slides in $3,3^{\prime}$-diaminobenzidine (DAB), followed by counterstained with hematoxylin, dehydrated in alcohol and xylene. Immunohistochemistry for other biomarkers, such as ER, PR, HER2 and Ki67 was done and evaluated in the department of Pathology at Shandong University by Roche Benchmark XT automated slide preparation system (Roche Ltd, Switzerland) according to the ultraview $\mathrm{DAB} v 3$ procedure.

Evaluation of immunohistochemical staining. The immunoreactivity was evaluated independently by two pathologists who were blinded with regard to the patient's clinical and pathological data. All of the slides were evaluated twice to confirm reproducibility of the results. Only invasive tumor cells were evaluated one each slide. The intensity of nuclear staining was scored and graded on a $0 \sim 3$ scale: 0 (negative); $1+$ (weak positive), $2+$ (moderate positive) and $3+$ (strong positive). This initial score was further classified as low expression $(0 \sim 1)$ or high expression $(2 \sim 3)$. For the evaluation of cyclinD1 staining, the cut-off point $10 \%$ was used for negative and positive group ${ }^{27}$. Tumors were considered positive for ER or PR if more than $10 \%$ tumor nuclei were immunostained ${ }^{28,29}$. The HER2 expression level was classified into four groups according to the scoring guidelines of the HercepTestTM ${ }^{30}$. Tumors were considered positive for HER2 gene if immunostaining was scored as $3+$ according to HercepTest criteria. The cut-off value of $15 \%$ for Ki67-positive cells was used according to our previous study for low proliferation and high proliferation tumors ${ }^{31}$.

Cell Culture and Small interfering RNA transfection. Human breast cancer cell line MDA-MB-231 and MCF-7 were obtained from Cell bank of Chinese Academy of Science (Shanghai, China) and cultured under conditions recommended by the American Type Culture Collection (Manassas, VA). MDA-MB-231 was cultured in L-15 medium (GIBCO) with 10\% fetal bovine serum (SAFC, BiosciencesTM), MCF-7 was cultured in DMEM media (HyClone) with $10 \%$ fetal bovine serum (SAFC, BiosciencesTM) and $0.05 \%$ insulin. They were all incubated at $37^{\circ} \mathrm{C}$ with $5 \% \mathrm{CO} 2$ except MDA-231 which is in non-O2 condition. Culture media were renewed every 2 days, and cells were passaged when they reached about $80 \%$ confluence using trypsinEDTA solution (Invitrogen Corp., Carlsbad, CA).

Three siRNAs was employed in order to deplete endogenous cellular hRad9 according to the manufacturer's instructions (Shanghai GenePharma Co, Ltd). hRAD9 siRNA 1 (sense:5'-CCAUCACUUUCUGCCUCAATT-3'; antisense: 5' UUGAGGCAGAAAGUGAUGGTT-3'), hRAD9 siRNA 2 (sense: $5^{\prime}$ - GCUGUAAGAUCCUGAUGAATT-3', antisense: 5' -UUCAUCAGGAUCUUACAGCTT-3'), hRAD9 siRNA 3 (sense: 5' - CGCCAUGGAAACCACUAUATT - $3^{\prime}$, antisense: 5' UAUAGUGGUUUCCAUGGCGTT - $3^{\prime}$, non-silencing control siRNA (sense: $5^{\prime}$ UUCUCCGAACGUGUCACGUTT-3'; antisense: $5^{\prime}$-ACGUGACACGUUCGGAGAATT- $3^{\prime}$ ). MCF-7 and MDA-MB-231 cells were seeded into a 6-well plate at $4 \times 10^{5}$ cells per well 24 hours before transfection. Cells were then treated with $20 \mathrm{uM}$ of either control or hRad9 siRNA using Lipofectamine 2000 reagent (1372416, invitrogen, USA)according to the manufacturer's protocol. The efficiency of cell transfection was assessed by counting the FAM-siRNA cells under fluorescence microscope. 48 hours after siRNA transfection, cells were collected for RT-qPCR and Western blot analysis to assess the RNA interfering efficiency.

RNA extraction and RT-qPCR. Total RNA was isolated using a Trizol reagent (Invitrogen, Carlsbad, CA) according to manufacturer's directions. cDNA was synthesized from $1 \mu \mathrm{g}$ of total RNA using Reverse Transcription Kit (Toyobo Co. Ltd., Osaka, Japan). The following primer sequences (Sangon Biotech, Shanghai Co. Ltd) were used: Hrad9 forward: 5' - TCTGCCTATGCCTGCTTTCTCT-3', reverse: 5'-AGCGGAAGACAGACAGGAAAGAC-3'; CHEK1 Forward: 5'-GGTCACAGGAGAGAAGGCAAT-3', reverse: 5' -TCTCTGAGCATCTGGTTCAGG-3'; GAPDH forward: 5'-AGAAGGCTGGGGCTCATTTG, reverse: 5' -AGGGGCCATCCACAGTCTTC-3'. RT-qPCR reaction was carried out by LightCycler 
(Bio-Rad, CFX96, USA) according to the manufacturer's instructions. Each sample was examined in triplicate and GADPH was used as the internal control. GraphPad Prism v5 (GraphPad Software, Inc., San Diego CA) was used to create the scatter plots. Data are representative of three independent experiments.

Western blot analysis. Adherent cells in the tissue culture wells were rinsed with PBS and directly lysed in the RIPA buffer. $50 \mu \mathrm{g}$ of cell lysate was fractionated on $12 \%$ SDSPAGE and transferred to polyvinylidene difluoride membrane (PVDF membrane). The PVDF membrane was blocked with $5 \%$ nonfat dry milk and probed with specific antibodies against hRad9 (1:5000, NBP1-47946, Novus Biologicals, LLC, USA) and chk1 (1:5000, NB100-464, Novus Biologicals, LLC, USA). Expression of GAPDH protein was assessed as an internal control by using anti-GAPDH antibody $(1: 200$, TA08, ZSGB-BIO, Beijing, China). Enhanced chemiluminescent detection (ECL) was performed according to the vendor's protocols (Santa Cruz Biotechnology).

Cytotoxicity assay. 24 hours after transfection, cells were transferred into 96-well plates and incubation for 12 hours. Cell viabilities were analyzed by MTS Cytotoxicity Assay Kit (Beyotime, Haimen, China) according to the manufacture's instruction. Briefly, 12 hours after incubation, the cells were treated with various concentrations of doxorubicin (0-10 uM ), After 48 hours, added $20 \mathrm{ul}$ of MTS solution to each well, and incubated for 3 hours followed by measuring at $560 \mathrm{~nm}$ in a Bio-Rad microplate reader (Hercules, CA, USA). The experiment was done in triplicate and repeated for at least 3 times.

Cell apoptosis assay. Cell apoptosis was detected using an annexin V-FITC/PI apoptosis detection kit (BB-4101-50T,Bestbio,China) according to the manufacturer's protocols. Briefly, cells were trypsinized with EDTA-free trypsin), and resuspended with $400 \mathrm{ul}$ annexin binding buffer at a concentration of $10^{6} \mathrm{cells} /$ $\mathrm{ml}$. Then, $5 \mathrm{ul} \mathrm{FITC-conjugated} \mathrm{AnnexinV} \mathrm{and} 10 \mathrm{ul} \mathrm{PI} \mathrm{were} \mathrm{added} \mathrm{to} \mathrm{the} \mathrm{cells} \mathrm{and}$ incubated at room temperature for 15 minutes in dark. Cell apoptosis assay was performed within $1 \mathrm{~h}$ post-staining on a flow cytometry.

Data analysis. Data were analyzed using SPSS 16.0 (SPSS Inc, IL, USA). Pearson's Chi-Square tests were applied to compare categorical variables and continuous variables were analyzed using $t$ test. Spearman's rank correlation test was used to evaluate correlations between variables. A two-tailed $\mathrm{P}$ value test was used in all analyses and a $\mathrm{P}$ value of $<0.05$ was considered statistically significant.

1. Banerji, S. et al. Sequence analysis of mutations and translocations across breast cancer subtypes. Nature 486, 405-409, doi:10.1038/nature11154 (2012).

2. Soerjomataram, I. et al. Global burden of cancer in 2008: a systematic analysis of disability-adjusted life-years in 12 world regions. Lancet 380, 1840-1850, doi:10.1016/S0140-6736(12)60919-2 (2012).

3. Lord, C. J. \& Ashworth, A. The DNA damage response and cancer therapy. Nature 481, 287-294, doi:10.1038/nature10760 (2012).

4. Bouwman, P. \& Jonkers, J. The effects of deregulated DNA damage signalling on cancer chemotherapy response and resistance. Nat. Rev. Cancer 12, 587-598, doi:10.1038/nrc3342 (2012).

5. Asakawa, H. et al. Prediction of breast cancer sensitivity to neoadjuvant chemotherapy based on status of DNA damage repair proteins. Breast Cancer Res. 12, R17, doi:10.1186/bcr2486 (2010).

6. Weinert, T. A. \& Hartwell, L. H. The RAD9 gene controls the cell cycle response to DNA damage in Saccharomyces cerevisiae. Science 241, 317-322 (1988).

7. Lieberman, H. B., Hopkins, K. M., Nass, M., Demetrick, D. \& Davey, S. A human homolog of the Schizosaccharomyces pombe rad9+ checkpoint control gene. Proc. Natl. Acad. Sci. U. S. A. 93, 13890-13895 (1996).

8. Volkmer, E. \& Karnitz, L. M. Human homologs of Schizosaccharomyces pombe rad1, hus1, and rad9 form a DNA damage-responsive protein complex. J. Biol. Chem. 274, 567-570 (1999).

9. Delacroix, S., Wagner, J. M., Kobayashi, M., Yamamoto, K. \& Karnitz, L. M. The Rad9-Hus1-Rad1 (9-1-1) clamp activates checkpoint signaling via TopBP1. Genes Dev. 21, 1472-1477, doi:10.1101/gad.1547007 (2007).

10. Ogston, K. N. et al. A new histological grading system to assess response of breast cancers to primary chemotherapy: prognostic significance and survival. Breast 12, 320-327 (2003).

11. Sorensen, C. S., Syljuasen, R. G., Lukas, J. \& Bartek, J. ATR, Claspin and the Rad9Rad1-Hus1 complex regulate Chk1 and Cdc25A in the absence of DNA damage. Cell cycle 3, 941-945 (2004).

12. Furuya, K., Poitelea, M., Guo, L., Caspari, T. \& Carr, A. M. Chk1 activation requires Rad9 S/TQ-site phosphorylation to promote association with C-terminal BRCT domains of Rad4TOPBP1. Genes Dev. 18, 1154-1164, doi:10.1101/ gad.291104 (2004).

13. Liu, S., Song, N. \& Zou, L. The conserved C terminus of Claspin interacts with Rad9 and promotes rapid activation of Chk1. Cell cycle 11, 2711-2716, doi:10.4161/cc.21041 (2012)

14. Didier, C. et al. G2/M checkpoint stringency is a key parameter in the sensitivity of AML cells to genotoxic stress. Oncogene 27, 3811-3820, doi:10.1038/ sj.onc.1211041 (2008).

15. Tse, A. N. et al. CHIR-124, a novel potent inhibitor of Chk1, potentiates the cytotoxicity of topoisomerase I poisons in vitro and in vivo. Clin. Cancer Res. 13, 591-602, doi:10.1158/1078-0432.CCR-06-1424 (2007).
16. Merry, C., Fu, K., Wang, J., Yeh, I. J. \& Zhang, Y. Targeting the checkpoint kinase Chk1 in cancer therapy. Cell cycle 9, 279-283 (2010).

17. Dai, Y. \& Grant, S. New insights into checkpoint kinase 1 in the DNA damage response signaling network. Clin. Cancer Res. 16, 376-383, doi:10.1158/10780432.CCR-09-1029 (2010).

18. Walton, M. I. et al. The preclinical pharmacology and therapeutic activity of the novel CHK1 inhibitor SAR-020106. Mol. Cancer Ther. 9, 89-100, doi:10.1158/ 1535-7163.MCT-09-0938 (2010).

19. Maniwa, Y. et al. Accumulation of hRad9 protein in the nuclei of nonsmall cell lung carcinoma cells. Cancer 103, 126-132, doi:10.1002/cncr.20740 (2005).

20. Cheng, C. K., Chow, L. W., Loo, W. T., Chan, T. K. \& Chan, V. The cell cycle checkpoint gene Rad9 is a novel oncogene activated by 11q13 amplification and DNA methylation in breast cancer. Cancer Res. 65, 8646-8654, doi:10.1158/00085472.CAN-04-4243 (2005).

21. Kebebew, E. et al. Diagnostic and prognostic value of cell-cycle regulatory genes in malignant thyroid neoplasms. World J. Surg. 30, 767-774, doi:10.1007/s00268005-0308-2 (2006).

22. Zhu, A., Zhang, C. X. \& Lieberman, H. B. Rad9 has a functional role in human prostate carcinogenesis. Cancer Res. 68, 1267-1274, doi:10.1158/00085472.CAN-07-2304 (2008).

23. Ormandy, C. J., Musgrove, E. A., Hui, R., Daly, R. J. \& Sutherland, R. L. Cyclin D1, EMS1 and 11q13 amplification in breast cancer. Breast Cancer Res. Treat. 78 323-335 (2003).

24. Holm, K. et al. Characterisation of amplification patterns and target genes at chromosome 11q13 in CCND1-amplified sporadic and familial breast tumours. Breast Cancer Res. Treat. 133, 583-594, doi:10.1007/s10549-011-1817-3 (2012).

25 . Mu, K. et al. Detection of CHK1 and CCND1 gene copy number changes in breast cancer with dual-colour fluorescence in-situ hybridization. Histopathology $\mathbf{5 8}$, 601-607, doi:10.1111/j.1365-2559.2011.03780.x (2011).

26. Verlinden, L. et al. The E2F-regulated gene Chk1 is highly expressed in triplenegative estrogen receptor/progesterone receptor/HER-2 breast carcinomas. Cancer Res. 67, 6574-6581, doi:10.1158/0008-5472.CAN-06-3545 (2007).

27. Rudas, M. et al. Cyclin D1 expression in breast cancer patients receiving adjuvant tamoxifen-based therapy. Clin. Cancer Res. 14, 1767-1774, doi:10.1158/10780432.CCR-07-4122 (2008).

28. Deyarmin, B. et al. Effect of ASCO/CAP guidelines for determining ER status on molecular subtype. Ann. Surg. Oncol. 20, 87-93, doi:10.1245/s10434-012-2588-8 (2013).

29. Iwamoto, T. et al. Estrogen receptor (ER) mRNA and ER-related gene expression in breast cancers that are $1 \%$ to $10 \%$ ER-positive by immunohistochemistry. J. Clin. Oncol. 30, 729-734, doi:10.1200/JCO.2011.36.2574 (2012).

30. Jacobs, T. W., Gown, A. M., Yaziji, H., Barnes, M. J. \& Schnitt, S. J. Specificity of HercepTest in determining HER-2/neu status of breast cancers using the United States Food and Drug Administration-approved scoring system. J. Clin. Oncol. 17, 1983-1987 (1999).

31. Li, L. et al. Genomic instability and proliferative activity as risk factors for distant metastases in breast cancer. Br. J. Cancer 99, 513-519, doi:10.1038/sj.bjc.6604479 (2008).

\section{Acknowledgments}

This work was supported by Grant from National Natural Science Foundation of China (30901739) and China Postdoctoral Science Foundation Grant (2013M531605).

\section{Author contributions}

K.M. designed the study, analyzed data, interpreted results, and drafted the manuscript. H.Q.Y. participated in the collection of clinical data, carried out the immunohistochemistry studies and interpreted results. R.R.S. carried out the siRNA studies, Western blot, and cytotoxicity test. Q.R.Y. participated in the design of the study and performed the statistical analysis. X.F.Z. participated in the design of the study and helped to interpreted results. X.C.Z. participated in cell culture. YW participated in immunohistochemistry studies. K.M. wrote the main manuscript text. H.Q.Y. prepared figures 1-2 and R.R.S. prepared figures 34. All authors reviewed the manuscript. All authors read and approved the final manuscript.

\section{Additional information}

Competing financial interests: The authors declare no competing financial interests.

How to cite this article: Yun, H. et al. Over expression of hRad9 protein correlates with reduced chemosensitivity in breast cancer with administration of neoadjuvant chemotherapy. Sci. Rep. 4, 7548; DOI:10.1038/srep07548 (2014).

This work is licensed under a Creative Commons Attribution-NonCommercialNoDerivs 4.0 International License. The images or other third party material in this article are included in the article's Creative Commons license, unless indicated otherwise in the credit line; if the material is not included under the Creative Commons license, users will need to obtain permission from the license holder in order to reproduce the material. To view a copy of this license, visit http:// creativecommons.org/licenses/by-nc-nd/4.0/ 